\title{
Accretion disc evolution in DW Ursae Majoris: A photometric study ${ }^{\star}$
}

\author{
V. Stanishev ${ }^{1,2}$, Z. Kraicheva ${ }^{1}$, H. M. J. Boffin ${ }^{3,4}$, V. Genkov ${ }^{1}$, C. Papadaki ${ }^{3}$, and S. Carpano ${ }^{5}$ \\ ${ }^{1}$ Institute of Astronomy, Bulgarian Academy of Sciences, 72, Tsarighradsko Shousse Blvd., 1784 Sofia, Bulgaria \\ e-mail: [zk;vgenkov]@astro.bas.bg \\ 2 Present address: Physics department, Stockholm University, Sweden \\ 3 Royal Observatory of Belgium, Avenue Circulaire 3, 1180 Brussels, Belgium \\ e-mail: [henri.boffin;Christina.Papadaki]@oma.be \\ ${ }^{4}$ European Southern Observatory, Karl-Schwarzschild-Str. 2, 85748 Garching-bei-München, Germany \\ 5 Institut für Astronomie und Astrophysik Universität Tübingen, Abteilung Astronomie, Sand 1, 72076 Tübingen, Germany \\ e-mail: carpano@astro.uni-tuebingen.de
}

Received 1 August 2003 / Accepted 4 December 2003

\begin{abstract}
We present an analysis of CCD photometric observations of the eclipsing novalike cataclysmic variable DW UMa obtained in two different luminosity states: high and intermediate. The star presents eclipses with very different depth: $\sim 1.2 \mathrm{mag}$ in the high and $\sim 3.4 \mathrm{mag}$ in the intermediate state. Eclipse mapping reveals that this difference is almost entirely due to the changes in the accretion disc radius: from $\sim 0.5 R_{\mathrm{L}_{1}}$ in the intermediate state to $\sim 0.75 R_{\mathrm{L}_{1}}$ in the high state $\left(R_{\mathrm{L}_{1}}\right.$ is the distance from the white dwarf to the first Lagrangian point). In the intermediate state, the entire disc is eclipsed while in the high state, its outer part remains visible. We also find that the central intensity of the disc is nearly the same in the two luminosity states and that it is the increase of the disc radius that is responsible for the final rise from the 1999/2000 low state. We find that the intensity profile of the disc is rather flat and suggest a possible explanation. We also discuss the effect of using a more realistic limb-darkening law on the disc temperatures inferred from eclipse mapping experiments. Periodogram analysis of the high state data reveals "positive superhumps" with a period of 0.1455 in 2002 and 0.1461 in 2003, in accord with the results of Patterson et al. However, we cannot confirm the quasi-periodic oscillations reported by these authors. We obtain an updated orbital ephemeris of DW UMa: $T_{\min }[\mathrm{HJD}]=2446229.00687(9)+0.136606527(3) E$.
\end{abstract}

Key words. accretion, accretion disks - stars: individual: DW UMa - novae, cataclysmic variables

\section{Introduction}

DW Ursae Majoris is an eclipsing nova-like (NL) cataclysmic variable $(\mathrm{CV})$ with an orbital period of $\sim 3.27 \mathrm{~h}$. The star is one of the NLs which show deep ( $\sim 4 \mathrm{mag}$ ) low states (Hessman 1990; Honeycutt et al. 1993, 2003), and "negative" and "positive superhumps" (Patterson et al. 2002). Shafter et al. (1988) carried out the first detailed photometric and spectroscopic study of DW UMa in a high state. The photometry revealed $\sim 1.5$ mag deep eclipses, with a peculiar V-like shape. The spectral observations showed single-peaked H, He I and He II emission lines with superimposed transient narrow absorption components. These peculiarities led Thorstensen et al. (1991) to group DW UMa along with SW Sex, V1315 Aql and PX And, and to define so-called SW Sex stars.

Send offprint requests to: V. Stanishev, e-mail: vall@astro.bas.bg

* based on observations obtained at Rozhen National Astronomical Observatory, Bulgaria, at Hoher List, Germany and at Kryoneri, Greece.
Biro (2000) presented $V$ and $R$ eclipse maps of DW UMa in a high state. The eclipse maps show that the radial profile of the accretion disc (AD) temperature distribution is rather flat, similarly to with what is found in other SW Sex novalikes (Rutten et al. 1992; Baptista et al. 1996). Biro (2000) also estimated the distance to the system to be $270 \pm 50 \mathrm{pc}$.

Spectral observations of Dhillon et al. (1994) in low state revealed narrow Balmer emission lines originating from the irradiated face of the secondary star. Marsh \& Dhillon (1997) could not detect the secondary star in their $I$-band spectra obtained in low state and derived a lower limit of the distance $450 \mathrm{pc}$ or $850 \mathrm{pc}$ depending on the assumed spectral type of the secondary. Ultraviolet spectroscopy during a recent low state was presented by Knigge et al. (2000). Surprisingly, they found that during the low state the continuum shortward of $1450 \AA$ was higher and bluer than in the high state. This led Knigge et al. (2000) to suggest that in the high state of DW UMa the white dwarf (WD) is permanently hidden from our sight by the rim of a flared accretion disc. Knigge et al. (2000) also modeled 
the ultraviolet spectrum of the WD and estimated the distance to DW UMa to be $830 \pm 150 \mathrm{pc}$.

Using the same observations as Knigge et al. (2000); Araujo-Betancor et al. (2003) could measure the eclipse phases of the WD. This allowed them to determine the system parameters of DW UMa with very good accuracy. Repeating the analysis of Knigge et al. (2000), and using $I$ - and $K$-band photometry, Araujo-Betancor et al. (2003) obtained two estimates of the distance to DW UMa: $930 \pm 150 \mathrm{pc}$ and $590 \pm 100$ pc, respectively.

In this article we present photometric observations of DW UMa in two different brightness states - intermediate and high. We performed periodogram analysis in the high state and detected "positive superhumps". We also present eclipse maps of DW UMa's accretion disc in the intermediate and high states. In the last part of the article we discuss the results and present our conclusions.

\section{Observations and data reduction}

Photometric CCD observations of DW UMa were obtained with the 2.0-m RCC telescope at Rozhen Observatory, the 0.85-m Schmidt telescope at the Royal Observatory of Belgium (ROB), the 1-m telescope at Hoher List Observatory and the 1.2-m telescope at Kryoneri Observatory between 2000 and 2003. Depending on the telescope and the atmospheric conditions, the exposure time used was between 20 and $60 \mathrm{~s}$. A journal of the observations is given in Table 1. The CCD frames were proceeded in the standard way with bias removal and flat-field correction, followed by aperture photometry with the DAOPHOT procedures (Stetson 1987). The Rozhen observations were performed with a Johnson $V$ filter, while the rest are unfiltered. For Rozhen observations the stars DW UMa-3 $(V=16.0)$ and DW UMa-2 $(V=17.043)$ from Henden \& Honeycutt (1995) served as a comparison and check, respectively. For the unfiltered observations as a comparison star we used the star GSC 3822 0070, whose standard magnitudes are given by Biro (2000). To be able to compare the $V$ photometry with the unfiltered one, we have corrected the unfiltered magnitudes in the following way. During the observations in year 2002 DW UMa was in a high state. Thus, we have computed the magnitude of GSC 38220070 in the instrumental system of the ROB Schmidt telescope so that the average outof-eclipse magnitude of DW UMa in that system to be equal to the average out-of-eclipse $V$ magnitude computed from the Rozhen data. We obtained $\sim 13.02$ which is just between the $V=13.29$ and $R=12.88$ mag estimated by Biro (2000). We further assumed that GSC 38220070 has the same magnitude in the system of the Hoher List 1-m telescope and corrected those magnitudes too. The Kryoneri photometry is relative to DW UMa-2. For DW UMa-2 we have obtained an unfiltered magnitude of $\sim 16.36$ and use it to put Kryoneri magnitudes in $V$. All the runs are shown in Fig. 1.

From Fig. 1 one can see that in 2001-2003 DW UMa was in its normal high state with out-of-eclipse magnitude $V \simeq 14.5$, but in 2000 we have caught the star on the rising branch from a deep low state. The later is more easily seen in Fig. 2 where we show part of the long-term light curve of DW UMa
Table 1. CCD observations of DW UMa.

\begin{tabular}{|c|c|c|c|}
\hline Date & $\begin{array}{c}\text { Start } \\
\text { HJD-2 } 450000\end{array}$ & $\begin{array}{c}\text { duration } \\
{[\mathrm{h}]}\end{array}$ & $\begin{array}{l}\text { mid-eclipse } \\
\text { [HJD] }\end{array}$ \\
\hline \multicolumn{4}{|c|}{ Rozhen 2-m, Johnson $V$ filter } \\
\hline 2000 Jan. 10 & 1554.34 & 7.13 & $\begin{array}{l}1554.33889 \\
1554.47524 \\
1554.61264\end{array}$ \\
\hline 2000 Jan. 13 & 1557.44 & 4.69 & $\begin{array}{l}1557.48099 \\
1557.61743\end{array}$ \\
\hline 2002 Jan. 11 & 2286.26 & 1.79 & 2286.27706 \\
\hline 2002 Jan. 13 & 2288.32 & 7.50 & $\begin{array}{l}2288.32641 \\
2288.46296 \\
2288.59911\end{array}$ \\
\hline 2002 Jan. 14 & 2289.40 & 4.44 & $\begin{array}{l}2289.41825 \\
2289.55524\end{array}$ \\
\hline 2002 Feb. 9 & 2315.25 & 9.95 & $\begin{array}{l}2315.37403 \\
2315.51110 \\
2315.64799\end{array}$ \\
\hline 2002 Feb. 17 & 2323.22 & 8.86 & $\begin{array}{l}2323.29749 \\
2323.43444 \\
2323.57099\end{array}$ \\
\hline \multicolumn{4}{|c|}{ ROB Schmidt telescope, unfiltered } \\
\hline 2000 Mar. 22 & 1626.38 & 5.50 & $\begin{array}{l}1626.46748 \\
1626.60419\end{array}$ \\
\hline 2002 Feb. 16 & 2322.39 & 3.96 & 2322.47774 \\
\hline 2002 Apr. 8 & 2373.45 & 4.24 & 2373.56879 \\
\hline 2002 Apr. 10 & 2375.38 & 4.43 & 2375.48054 \\
\hline 2002 Apr. 24 & 2389.38 & 4.63 & $\begin{array}{l}2389.41500 \\
2389.55209\end{array}$ \\
\hline 2003 Mar. 16 & 2715.52 & 2.68 & \\
\hline 2003 Mar. 22 & 2721.51 & 4.23 & 2721.64166 \\
\hline 2003 Apr. 15 & 2745.38 & 5.00 & $\begin{array}{l}2745.41090 \\
2745.54838 \\
\end{array}$ \\
\hline \multicolumn{4}{|c|}{ Hoher List 1-m, unfiltered } \\
\hline 2000 Feb. 10 & 1585.50 & 3.26 & 1585.62166 \\
\hline 2000 Feb. 11 & 1586.60 & 3.80 & 1586.71482 \\
\hline 2001 Jan. 15 & 1925.37 & 4.69 & 1925.49886 \\
\hline 2001 Jan. 16 & 1926.58 & 4.64 & $\begin{array}{l}1926.59172 \\
1926.72804\end{array}$ \\
\hline 2003 Mar. 04 & 2703.44 & 1.52 & 2703.47304 \\
\hline 2003 Mar. 06 & 2705.34 & 8.31 & $\begin{array}{l}2705.52281 \\
2705.65868\end{array}$ \\
\hline 2003 Mar. 07 & 2706.30 & 7.47 & 2706.34213 \\
\hline \multicolumn{4}{|c|}{ Kryoneri $1.2-\mathrm{m}$, unfiltered } \\
\hline 2003 Jun. 02 & 2793.31 & 2.89 & 2793.36068 \\
\hline
\end{tabular}

obtained by RoboScope (Honeycutt et al. 2003) with the epochs of our observations marked with the vertical bars. In Fig. 2 the open squares show the nightly mean out-of-eclipse magnitude of DW UMa during our observations. It is seen that our observations follow the photometry of Honeycutt et al. (2003) very well. This also gives us confidence in the correction of the unfiltered observations described above.

Figure 1 also reveals the huge difference in the eclipse depth in 2001-2003 and Jan. 2000: 1.2 and 3.4 mag, respectively. To our knowledge the eclipses we see in 2000 are among the deepest ever observed in a CV. The only close example 

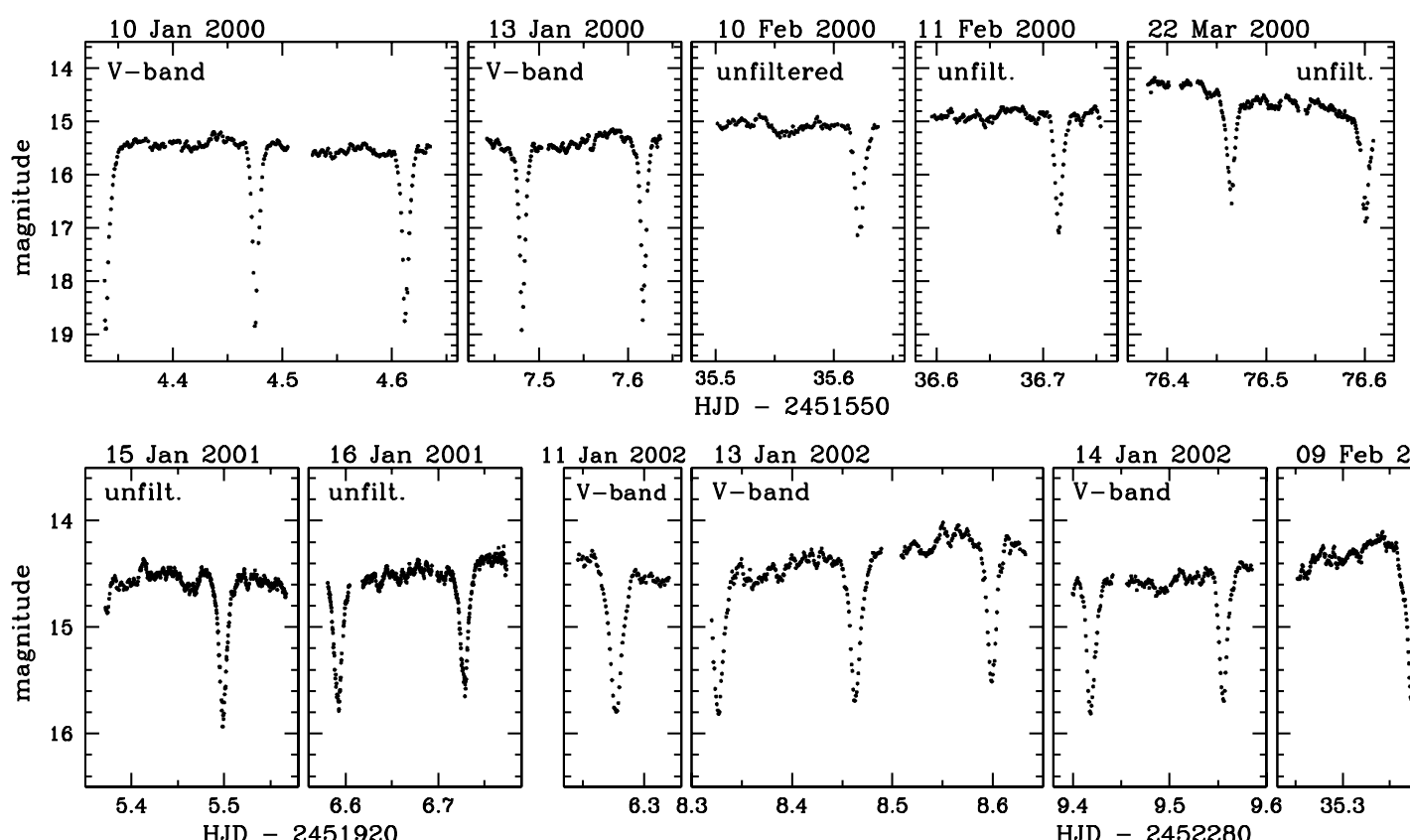

11 Jan $2002 \quad 13$ Jan 2002
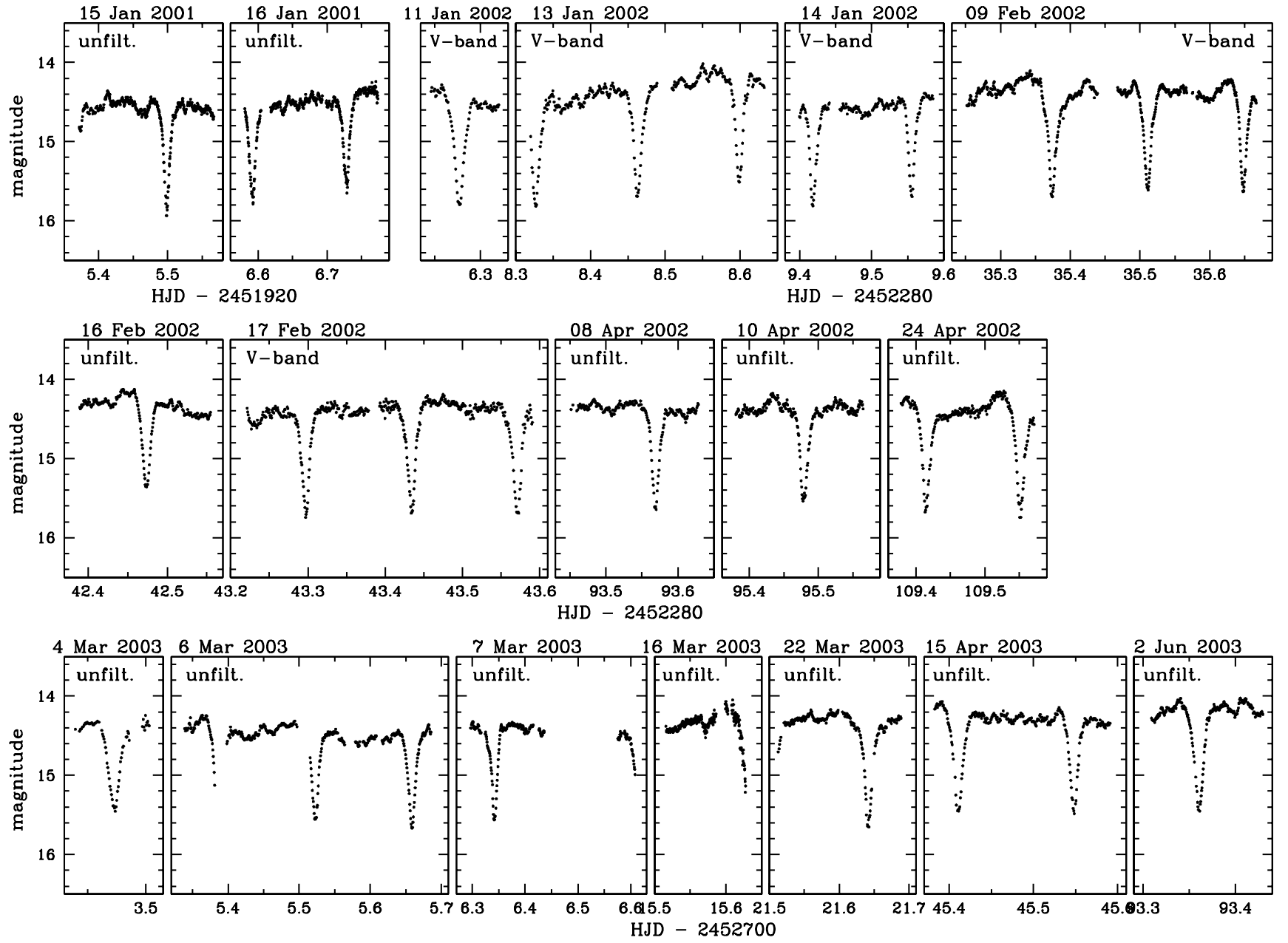

Fig. 1. Photometric observations of DW UMa. Note the much larger range of the $y$-axis in the upper row panel.

is GS Pav, which sometimes also shows $\sim 3.2-3.3$ mag deep eclipses (Groot et al. 1998). Figure 3 shows the dependence of the eclipse depth on the magnitude at orbital phase zero. The later was estimated by fitting low-order polynomial to the outof-eclipse measurements before and after the given eclipse. The dependence is almost linear and the eclipse depth decreases with system luminosity.

\section{Results}

\subsection{The orbital ephemeris}

The eclipse timings listed in Table 1 were determined by fitting parabolas to the lower half of the eclipses. These timings were analyzed together with those given by Dhillon et al. (1994); Biro (2000); Biro \& Borkovits (2000) and Borkovits et al. $(2001,2002,2003)$ to obtain the following orbital ephemeris:

$$
T_{\min }[\mathrm{HJD}]=2446229.00687(9)+0.136606527(3) E,
$$

that is very close to that derived by Biro (2000). The rms around the fit is $\sim 31 \mathrm{~s}$. We note that Araujo-Betancor et al. (2003) used a different ephemeris, which left them with $(\mathrm{O}-\mathrm{C})$ residuals of +73 s. We note that our ephemeris (and that of Biro 2000) nearly corrects for this residuals. 


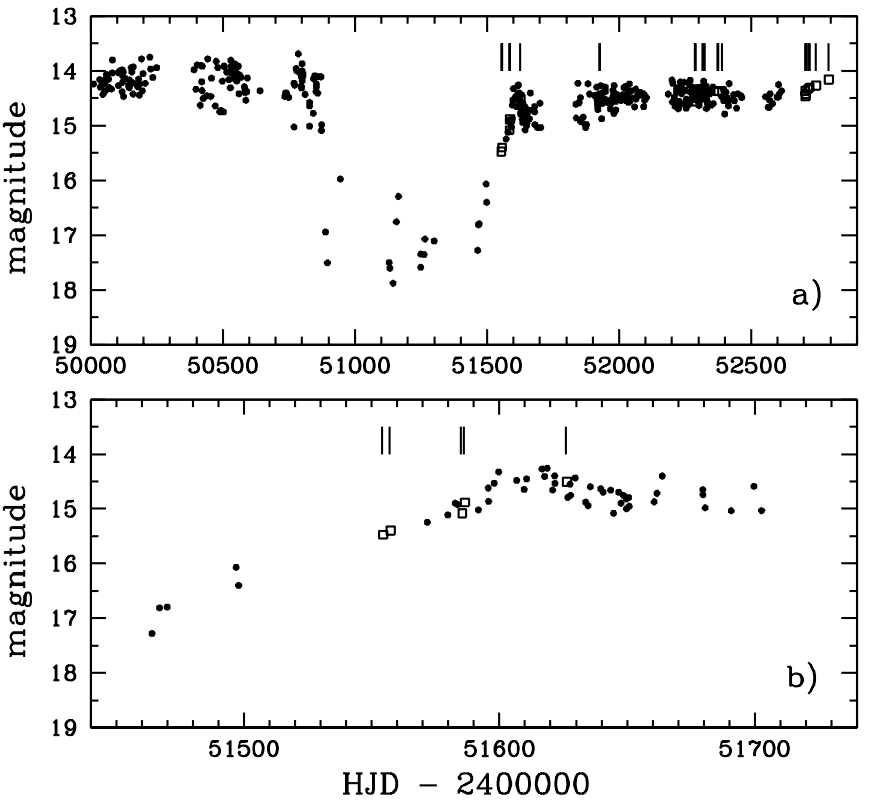

Fig. 2. Part of the long-term light curve of DW UMa obtained by RoboScope (Honeycutt et al. 2003) with the epochs of our observations marked with the vertical bars and the mean out-of-eclipse magnitudes shown with open squares. Our observations in 2000 have been obtained on the rising branch of a deep low state. For clarity, the RoboScope data obtained during eclipse have been removed.

\subsection{Periodogram analysis}

After removing the measurements during the eclipses from the light curves, the data obtained in 2002 and 2003 were searched for the "positive superhumps" reported by Patterson et al. (2002). The periodogram of the "raw" light curves did not give any conclusive results. The most likely reason is the combination of very complex light curves, removing $\sim 1 / 3$ of the data around eclipse and the sparse distribution of the observations. We would like to note one peculiarity of the DW UMa light curves: in some of the runs we clearly see a linear increase/decrease of the system brightness (22 March 2000, 16 Jan. 2001, 13 and 14 Jan. 2002). The same seems to be also present in the observations of Biro (2000; see his Fig. 1) too. This suggests that DW UMa's mean magnitude varies on time scale of $\sim 1-2$ days. We note that a 2.2 days (in 2002) or 2.1 days (in 2003) periodicity could be linked to the superhump we have detected in the light curves. It is however still premature to claim that such a periodicity as been found. From our Figs. 1 and 3, and Biro's Fig. 1, one can see that these brightness variations are not accompanied by variations of the eclipse depth. This suggests a global brightening of the AD without significant changes of its brightness distribution or radius.

To account for these trends we fitted straight line to all the runs, subtracted it from them and repeated the periodogram analysis. To de-convolve the computed power spectrum with the spectral window, the CLEAN algorithm of Roberts et al. (1987) has been used. The results are presented in Fig. 4 and show that in 2002 data we could detect "positive superhumps" with a period of $P_{\mathrm{sh}}^{+}=0.1455$, which is very close to that of Patterson et al. (2002). We also see two of the other

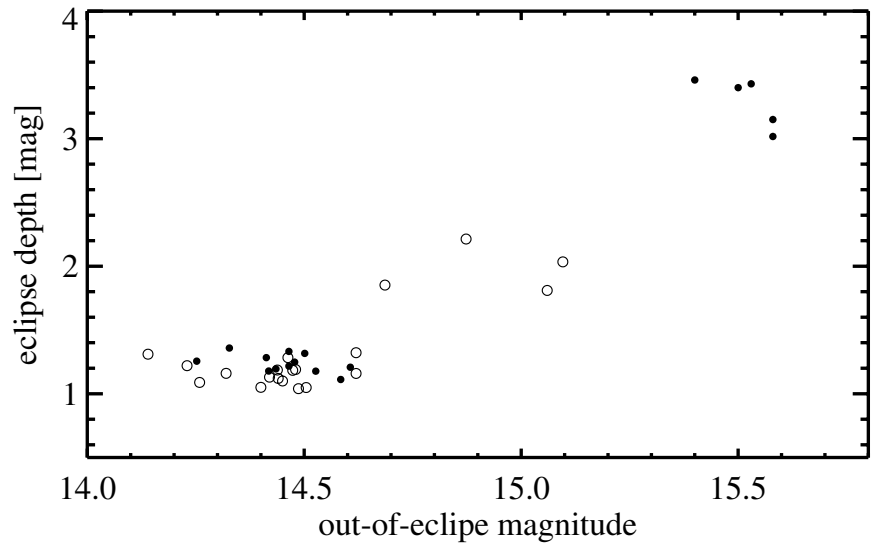

Fig. 3. Eclipse depth as a function of the out-of-eclipse magnitude of DW UMa. Filled circles - Rozhen $V$-band photometric observations; open circles - unfiltered observations.

three peaks reported by Patterson et al. (2002), those close to the first harmonics of the orbital and "positive superhumps" periods. The multi-sinusoidal fit to the data gives the following full amplitudes of these modulations: 0.096, 0.053 and 0.044 mag, in order of decreasing period. In 2003 we also detect "positive superhumps" with a period of $P_{\mathrm{sh}}^{+}=0.1461$ but none of the shorter signals. The full amplitude of the modulation is $\sim 0.08$ mag.

We also searched for periodic and quasi-periodic oscillations (QPOs) on the minute time scale. The presence of QPOs with characteristic periods in the range 1000-4000 s in NLs is now a well established observational fact (for a short review see Patterson et al. 2002). Patterson et al. (2002) also see in a few SW Sex stars, including DW UMa, stable oscillations with periods in the same range as the QPOs. Figure 5 shows the mean power spectrum of DW UMa runs in double logarithmic scale. The power spectrum has a typical "red noise" shape with a power-law decrease of the power with frequency $P(f) \propto f^{-1.59 \pm 0.02}$ and shows no traces of QPOs. We also do not see the stable oscillations with periods of $2375 \mathrm{~s}$ and $2974 \mathrm{~s}$ reported by Patterson et al. (2002). The "red noise" shape of the power spectrum is believed to be a manifestation of the flickering, which is strong in DW UMa and as usual appears as well defined peaks with a typical duration of 5-15 min, and amplitudes reaching $\sim 0.2-0.3 \mathrm{mag}$. The standard deviation of the light curves after subtraction of the best fit is $\sim 0.06 \mathrm{mag}$, which is consistent with what is seen in other NLs (Kraicheva et al. 1999a, 1999b; Stanishev et al. 2002).

We also note that in some of the runs, 2 Feb. 2002 and 24 Apr. 2002 for example, a hump just before the eclipse is clearly seen. Such humps are commonly observed in highinclination dwarf novae in quiescence and are caused by the hot spot formed where gas stream hits the outer edge of the disc. The spot also makes the eclipse to appear asymmetric, with a well pronounced shoulder on the egress. The eclipses of DW UMa, including these with preceding humps are fairly symmetric and with no hints of hot spot. We believe that the "pre-eclipse humps" seen in DW UMa are a result of superhumps in conjunction with strong flickering activity. 

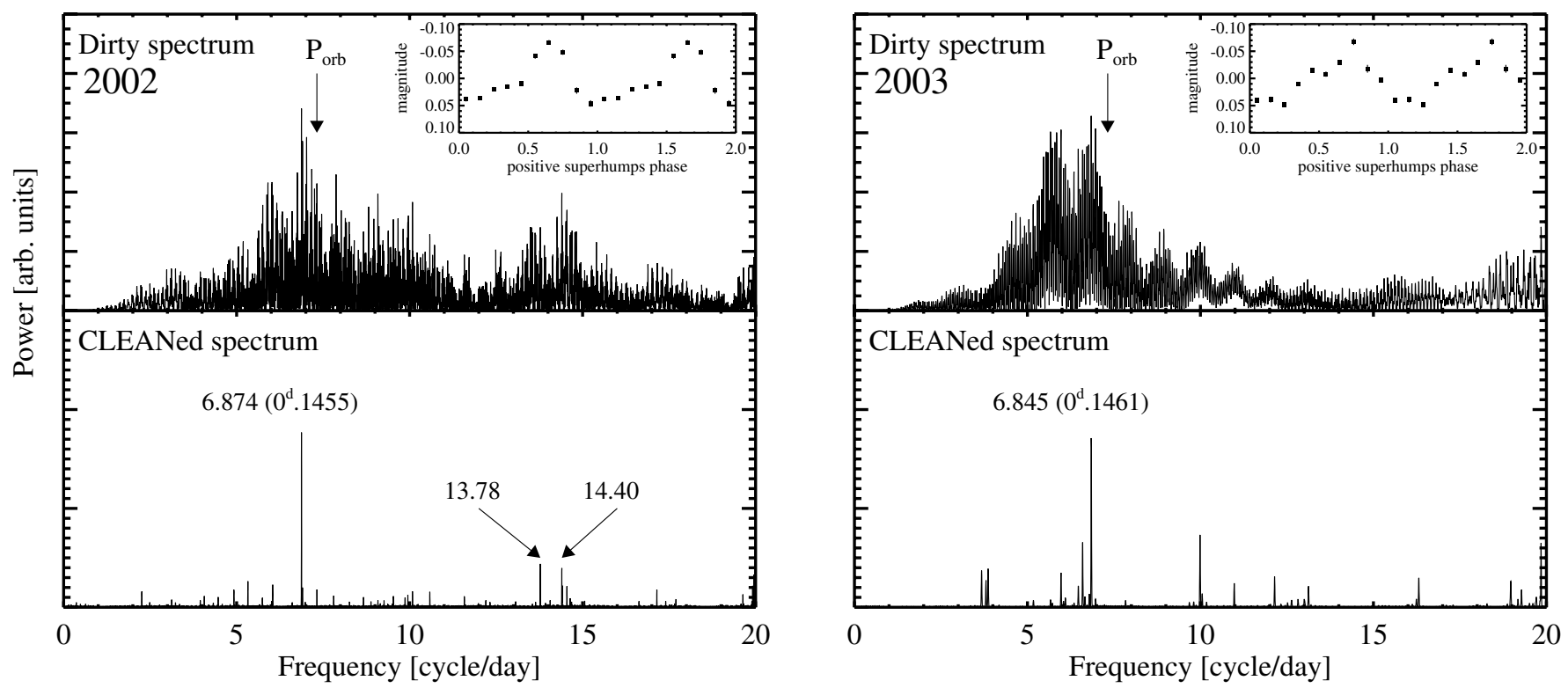

Fig. 4. Periododgram analysis of DW UMa in 2002 - left and in 2003 - right. The insets show the mean superhump shape.

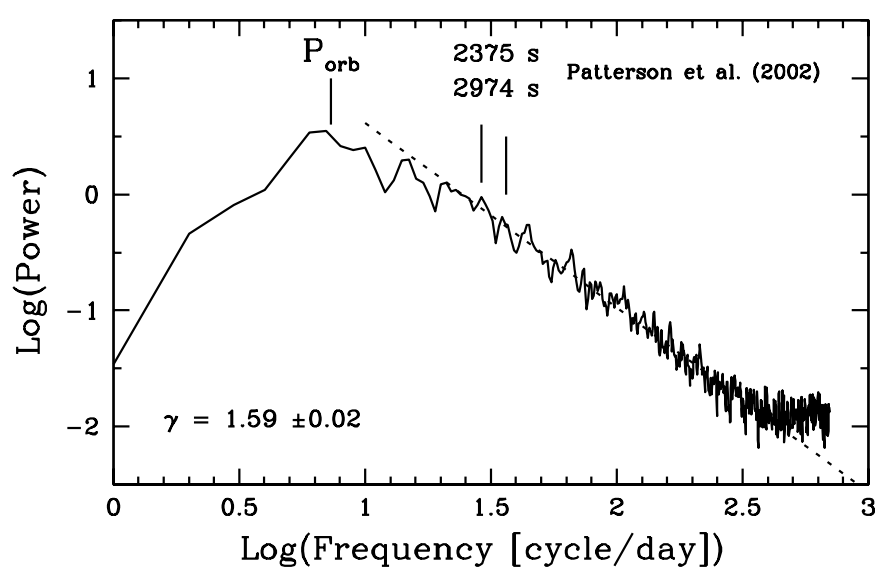

Fig. 5. Mean power spectrum of DW UMa in double logarithmic scale.

\subsection{Eclipse mapping}

The eclipse mapping method (Horne 1985) and the Maximum Entropy technique (Skilling \& Bryan 1984) was used to recover the surface brightness distribution of the accretion disc in DW UMa. The code we use (written by one of us - V.S.) allows for the presence of light that is never eclipsed. We assume a flat disc which lays in the orbital plane. Eclipse mapping assumes that all brightness variations result from the eclipse of the disc by the secondary star. Thus, any brightness variations due to anisotropic emission from the hot spot or superhumps cannot be handled by the algorithm. To account for these brightness variations we fitted the out-of-eclipse flux with a low-order polynomial function and normalized the eclipse by the fit.

The shape of the individual eclipses is highly variable, in particular in the upper half of the profile. This is most likely due to the flickering and is not related to real changes of the AD structure. As mentioned before, flickering in DW UMa is strong and certainly can affect the upper part of the eclipse profile. To reduce the influence of flickering and other noise the eclipses were averaged in small phases bins. We note that even if some of the "pre-eclipse humps" discussed above are caused by temporary appearance of a hot spot and in these cases the eclipse shape variations are not caused by flickering, when working with an average eclipse the spot will not be detected in the eclipse maps. Because of the large difference in the eclipse depth we have analysed the eclipses in the high and intermediate state separately. In addition, the $V$-band and the unfiltered high state eclipses were also analysed separately. In the high state we have enough eclipses to try to reduce the influence of the flickering even more: we averaged only the lowest $70 \%$ of the point in each bin. Such an approach is reasonable because flickering increases the observed flux. The error bars assigned to the mean values are the standard errors of the mean. The mean eclipses are shown in Fig. 6a. They have been scaled so as to have out-of-eclipse flux equal to the mean flux at orbital phase zero. The mean fluxes at orbital phase zero were determined from the fits used to normalize the eclipses.

To use the eclipse mapping method one needs to know the orbital inclination $i$ and the mass ratio $q=M_{2} / M_{1}$, where $M_{1}$ is the mass of the WD and $M_{2}$ is the mass of the secondary star. In an eclipsing $\mathrm{CV}, i$ and $q$ are related trough the duration of the eclipse of the WD $\Delta \phi=\phi_{\mathrm{e}}-\phi_{\mathrm{i}}$, where $\phi_{\mathrm{i}}$ and $\phi_{\mathrm{e}}$ are the ingress and egress phases of the WD's center. In NLs, $\phi_{\mathrm{i}}$ and $\phi_{\mathrm{e}}$ can only be directly measured in the low state. AraujoBetancor et al. (2003) obtained UV observations of DW UMa in the low state and could measure $\phi_{\mathrm{i}}$ and $\phi_{\mathrm{e}}$. This allowed them to tightly constrain the system parameters. More specifically they obtained $q=0.39 \pm 0.12$ and $i=82^{\circ} \pm 4^{\circ}$, and we used these values in our eclipse mapping analysis ${ }^{1}$.

1 The detection of "positive superhumps" in DW UMa indicates a lower value of $q \sim 0.30-0.33$. This is well in the $1 \sigma$ range given by Araujo-Betancor et al. (2003). We tried eclipse mapping with $q=0.3$, but this did not change the results significantly. The results presented below are obtained with $q=0.39$. 

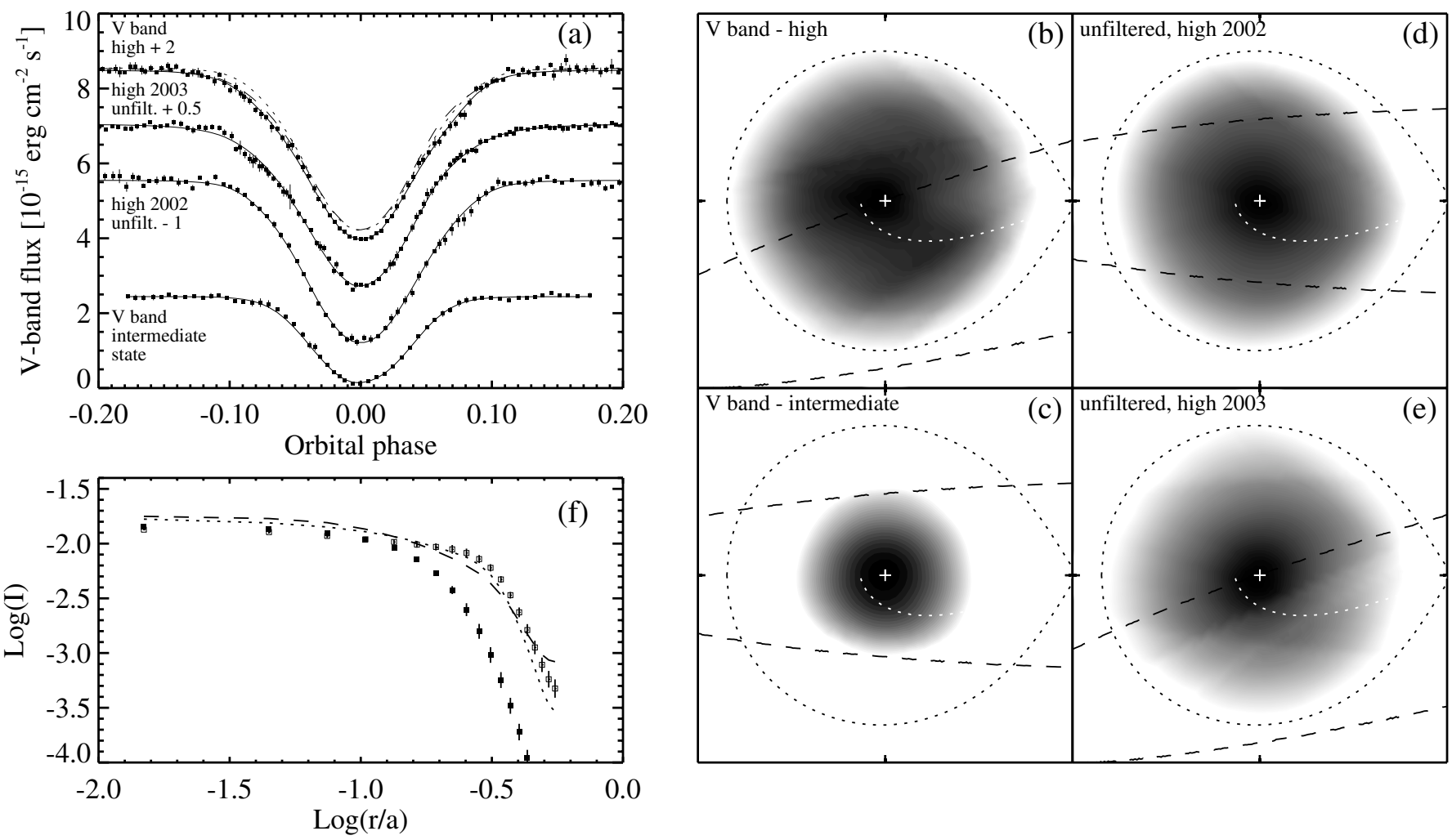

Fig. 6. Eclipse mapping of DW UMa. a) mean light curves with the best fits. The dotted and the dashed lines are the fits to the unfiltered high state eclipses in 2002 and 2003, respectively, overplotted on the $V$-band high state one; b-e) accretion disc eclipse maps, the WD's Roche lobe is shown with dotted line and the shadow of the secondary at orbital phases 0.0 and 0.041 with dashed line; f) radial intensity profiles of the accretion disc: open squares $-V$-band high state, filled squares $-V$-band intermediate state, and dotted and dashed lines - unfiltered high state in 2002 and 2003, respectively.

In certain circumstances $\Delta \phi$ can be also measured from high state eclipses (Wood et al. 1992). Such measurements rely on the fact that in certain combinations of $i$ and $q$, at $\phi_{\mathrm{i}}$ and $\phi_{\mathrm{e}}$ approximately half of the disc area is eclipsed. This is the case for most deeply eclipsing systems, such as DW UMa, provided that $q \geq 0.3$ and $i \geq 80^{\circ}$. Thus, $\Delta \phi$ can be measured from the points of half-intensity of the eclipse. In almost all NLs this turns out to be the only method available to measure $\Delta \phi$. However, the accuracy of these measurements is usually very low, and DW UMa itself is a good example. Published values of $\Delta \phi$ measured from high state eclipses range from $\sim 0.073$ (Shafter et al. 1988) to $\sim 0.090$ (Dhillon et al.1994; Biro 2000). From our high state eclipses we determine $\Delta \phi \sim 0.064-0.068$, the lowest values ever measured in DW UMa. The only measurements of $\Delta \phi$ close to the accurate value of Araujo-Betancor et al. (2003) have been obtained when the AD contribution is small, $\sim 0.083$, by Dhillon et al. (1994) in the low state. From the intermediate state eclipses we obtained $\Delta \phi \simeq 0.080$, which is also very close to the value of Araujo-Betancor et al. (2003). It is clear then that measurements of $\Delta \phi$ from eclipses in the high state and system parameters determinations based on these have to be interpreted with extreme caution.

The eclipses are rather symmetric, which suggests a nearly symmetric distribution of the AD intensity. That is why in the reconstructions we used a "full azimuthal streaming" default image (Horne 1985). The eclipses were fitted to reduced $\chi^{2}=1$ and the fits are shown with solid lines in Fig. 6a. The corresponding eclipse maps are shown in logarithmic gray-scale in Figs. 6b-e. The outermost disc parts displayed correspond to the level where the intensity drops below $10 \%$ of the maximum, which we adopt as measure of the disc radius. The corresponding radial profiles of the disc images are shown in Fig. 6f. The main results of the eclipse mapping of DW UMa can be summarized as follows:

- the AD radius increased from $\sim 0.5 R_{\mathrm{L}_{1}}$ in the intermediate state to $\sim 0.75 R_{\mathrm{L}_{1}}$ in the high state $\left(R_{\mathrm{L}_{1}}\right.$ is the distance from the WD to the first Lagrangian point $\mathrm{L}_{1}$ );

- the AD brightness distribution in both states is very flat;

- the central intensities of the high and intermediate state maps are very close (Fig. 6f). Thus, it seems that the increase of the AD radius can fully account for the long-term brightness variations, at least for the last one magnitude on the rising part of the 1999 low state;

- in the high state a significant amount of uneclipsed light is detected: $\sim 9 \%$ in the $V$-band and $\sim 17 \%$ in the unfiltered maps. The uneclipsed light is essentially zero in the intermediate state;

- in the intermediate state the $\mathrm{AD}$ is almost totally eclipsed, whereas in the high state, a large part of the disc remains visible at mid-eclipse. This is the reason for the large difference in the eclipse depth. 


\section{Discussion}

Perhaps the most intriguing results of this study are the AD radius variations and the nearly equal central intensities of the high and intermediate state maps. As high and low states are caused by strong variations of mass accretion rate, the later is difficult to explain within the standard accretion disc theory. However, what we formally call "intermediate state" is in fact a transition from the 1999/2000 low state to the high state and during this transition the disc might not be in equilibrium with the actual accretion rate. However, this is most likely not the case. Figure $2 b$ shows that the recovery from the low state is rather slow and takes $\sim 4$ months, i.e. the disc has enough time to adjust its structure to changes of mass accretion rate. This suggests that at given value of the accretion rate some kind of saturation occurs and further increase of the accretion rate does not lead to increase of the emission from the inner part of the disc. Instead, the rise of the system luminosity is due to an increase of the disc radius from $\sim 0.5 R_{\mathrm{L}_{1}}$ to $\sim 0.75 R_{\mathrm{L}_{1}}{ }^{2}$. These values are in accord with the AD radii in NLs, including DW UMa itself, determined by Harrop-Allin \& Warner (1996) from highand low-state observations. Note that the ratio of the area of circles with these radii is $\sim 2.25$. If the AD brightness distribution does not depend on radius, this will give $\sim 1$ mag increase of the brightness, as observed. The reason of this behavior is not clear. It could be that at certain value of the accretion rate some mechanism starts removing energy from the inner disc, thus preventing further increase of the emission of this part. Thus, it appears that this issue could be tightly related to the mechanism responsible for the flat intensity profile of the disc, which we discuss next.

The flat AD intensity profile found in SW Sex type NLs is still a puzzle. This problem has been extensively discussed in the literature, but there is no widely accepted model. Currently, two of the proposed models seems to be most viable. Accretion disc wind (or something else) can remove energy from the inner part of the disc thus causing the profile to be flatter than the steady-state $T_{\text {eff }} \propto r^{-3 / 4}$ dependence. The second model assumes that SW Sex stars are intermediate polars and that the inner part of their discs is missing. In order to account for the other properties of the SW Sex stars it is also assumed that after the first impact with the AD's edge, part of the gas in the accretion stream continues moving above the disc and hits its surface again close to the WD. In the intermediate polar model the stream hits the WD's magnetosphere (for a review see Hellier 2000). In this later model the flat profiles found in the eclipse mapping experiments are simply a result of the assumption that the AD extends down to the WD's surface. The later model has recently gained additional support. Rodriguez-Gill et al. $(2001,2001)$ reported the discovery of periodic circular polarization in two SW Sex stars. Patterson et al. (2002) reported possible detections of periodic signals $(\sim 1000 \mathrm{~s})$ in the light curves of several SW Sex and suggested that this could be a manifestation of the rotation of a highly magnetic WD. Note, however, that in the intermediate polars the radius of the

\footnotetext{
${ }^{2}$ We stress that the fact that we see this behavior on the rising branch of the low state does not necessarily mean that one should expect the opposite on the falling branch.
}

WD's magnetosphere or equivalently the inner radius of the AD $r_{\text {in }}$ depends on the mass accretion rate $\dot{M}: r_{\text {in }} \propto \dot{M}^{-2 / 7}$. Thus, the higher $\dot{M}$ the lower $r_{\text {in }}$. Therefore, it can be expected that in the high-state eclipse mapping analysis the AD brightness distribution will appear closer to the steady-state law than that in intermediate state. We do not see this behavior and the AD brightness distribution is rather flat in both state eclipse maps.

Recently, Knigge et al. (2000) found that during the 1999 low state, the UV continuum of DW UMa shortward of $1450 \AA$ was higher and bluer than in the high state. The authors suggest that in the high state the WD and the inner hot part of the disc are permanently hidden from our sight by the rim of a flared AD. This certainly can alter the disc intensity profile of DW UMa and cause it to appear flat in the eclipse maps. However, the weakness of this model is the need of a very high AD rim; its height should be at least $\sim 0.15 R_{\text {disc }}$ if the system inclination is $82^{\circ}$. With the present knowledge, it is difficult to understand how such a high rim can be formed. Moreover, it should have a constant height because Knigge et al. (2000) found that in the low state the UV spectrum is at all orbital phases bluer and the flux higher than in high state. In the classical steady-state AD model this would require unrealistically high mass accretion rate. The impact of the accretion stream with the disc can form a thick bulge but it could not persist along the whole disc rim.

Here, we suggest another possibility. Bobinger et al. (1997) found that the intensity profile of IP Peg's AD in decline from outburst is flat. The authors explored many possible causes of this and concluded that the presence of an optically thick and geometrically flat layer on top of the AD's surface masks its true intensity profile. Bobinger et al. (1997) suggested that this layer may be formed by the wind from the disc. This is even more relevant in NLs, which generally have hotter discs than dwarf novae.

Knigge \& Drew (1997) modeled the wind in the NL UX UMa and found that between the disc's photosphere and fastmoving part of the wind there is a transition zone of a slowly outflowing and relatively dense layer. We suggest that such a layer may also be present in DW UMa, and may shade the WD and the inner hot part of the disc, or what is more likely, attenuate the radiation of the inner disc and the WD. Although this may seem somewhat speculative, it can account for (1) the flat disc profile; (2) the red UV spectrum in high state and (3) nearly the same temperature of the inner disc in the high and intermediate states. However, it should be borne in mind that in UX UMa itself this layer does not flatten the AD's temperature profile; UX UMa is often given as an example where the results of eclipse mapping are consistent with the steady-state $T_{\text {eff }} \propto r^{-3 / 4}$ law. The reason for the difference may be different parameters of the wind.

Having the eclipse map calculated one can estimate the brightness temperature $T_{\mathrm{BR}}$ of the accretion disc assuming blackbody emission. This is done for the $V$-band eclipse maps by solving for $T_{\mathrm{BR}}$ the following equation:

$f_{j}=\frac{C \delta_{j} \cos i}{D^{2}} \frac{\int v(\lambda) B_{\lambda}\left(T_{\mathrm{BR}, j}\right) \lambda \mathrm{d} \lambda}{\int v(\lambda) \lambda \mathrm{d} \lambda}$ 

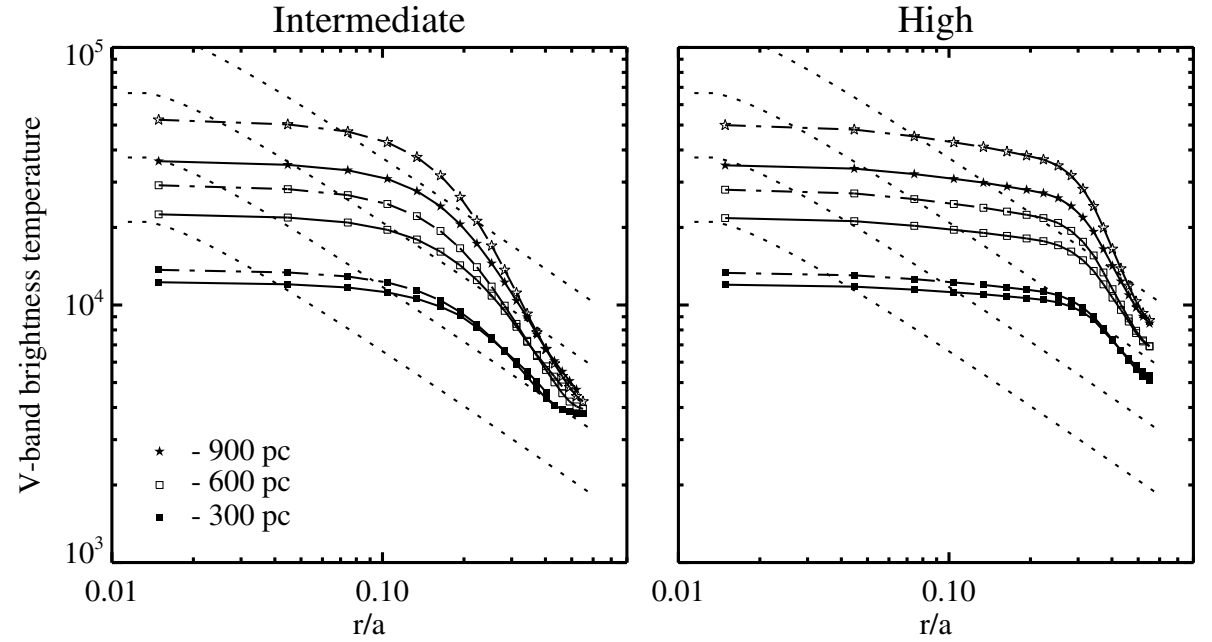

Fig. 7. Radial profile of the AD brightness temperature obtained by using (i) temperature dependent limb-darkening coefficient symbols connected with solid lines and (ii) with constant limb-darkening coefficient of 0.6 - symbols connected with dash-dotted lines. The dotted curves show the effective temperature of steady-state ADs for mass transfer rates $\dot{M}=10^{-10}-10^{-7} M_{\odot} \mathrm{yr}^{-1}$ onto $0.77 M_{\odot}$ WD.
Here $f_{j}$ is the flux emitted by the $j$ th element of the $\mathrm{AD}, T_{\mathrm{BR}, j}$ and $\delta_{j}$ are its brightness temperature and area, respectively, $B_{\lambda}\left(T_{\mathrm{BR}, j}\right)$ is the blackbody spectral distribution, $v(\lambda)$ is the response of the $V$ bandpass and $D$ is the distance to the system. As noted by Baptista et al. (1998) the relation between the brightness temperature and the effective temperature is not trivial and to obtain such a relation the vertical structure of the disc has to be known. However, the emission of the hot optically thick ADs in novalikes probably closely follows the blackbody law and $T_{\mathrm{BR}}$ should be close to the effective temperature. Optically thick ADs are however subject to an effect analogous to the limb-darkening in stars and the factor $C$ takes this into account. This later effect greatly complicates the situation because it is well known that 1) the real limb-darkening differs significantly from often used linear law and 2) $C$ is a function of surface gravity, temperature and wavelength (Wade \& Rucinski 1985; Diaz et al. 1996).

To investigate the importance of limb-darkening further, we have calculated $C$ by using the available synthetic stellar spectra with solar abundances and $\log g=5$ calculated by Kurucz ${ }^{3}$. We calculated $C$ for $V$-band wavelengths, $i=82^{\circ}$ and temperatures from 5000 to $50000 \mathrm{~K}$. Not surprisingly $C$ turns out to depend strongly on the temperature (non-linearly) and changes from $\sim 0.35$ at $T_{\text {eff }}=5000 \mathrm{~K}$ to $\sim 0.84$ at $T_{\text {eff }}=50000 \mathrm{~K}$. Thus, it seems that the dependence of limb-darkening on the temperature can affect significantly the estimation of AD brightness temperature obtained from eclipse mapping. This dependence would also affect the obtained radial profile of the temperature in ADs with temperature varying in a wide range. This would have a direct consequence on the inferred accretion rates and distances to the CVs studied by eclipse mapping.

Although ADs structure is different from that of the normal stars, Diaz et al. (1996) have shown that the difference between limb-darkening laws obtained with Kurucz stellar spectra and AD model calculations is small (see Fig. 1 in Diaz et al. 1996). Thus, the main source of error when calculating $C$ is the assumption of constant surface gravity $\log g=5(\log g=5$ is the maximal gravity in Kurucz' models). In real discs the gravity changes from $\log g \sim 4.5$ at the outer edge to $\log g \sim 6.5-7.0$

\footnotetext{
3 Available at http://cfaku5.harvard.edu
}

at the inner parts. The work of Wade \& Rucinski (1985) shows that in stars for given effective temperature the limb-darkening coefficient decreases with increasing $\log g$, which means $C$ increases with $\log g$. If this holds true for ADs and $\log g>5$, then the temperatures one obtains with $\log g=5$ overestimate the real temperature of the $\mathrm{AD}$, particularly in its inner part, where $\log g>5$; the degree of overestimation is however difficult to estimate without model spectra with $\log g>5$.

Since the distance to DW UMa is rather uncertain (see the Introduction for details) we have solved Eq. (2) for three distances: 300, 600 and 900 pc. Figure 7 shows the results: symbols connected with solid lines show solutions with limbdarkening as determined from Kurucz' model spectra, and those connected with dash-dotted lines show the brightness temperature obtained using a linear limb-darkening law with a coefficient of 0.6. Also shown with dotted curves is the expected effective temperature radial distribution of steady-state ADs for mass transfer rates $\dot{M}=10^{-10}-10^{-7} M_{\odot} \mathrm{yr}^{-1}$ onto $0.77 M_{\odot}$ WD (Araujo-Betancor et al. 2003). It is clearly seen that the temperature of the AD in DW UMa in both high and intermediate state is very far from following the steady-state radial dependence: it is much flatter that expected. This finding is in accord with the results of Biro (2000). Also seen is that the usage of more realistic limb-darkening law results in lower AD temperatures. The effect on the radial dependence is however rather small, which is a direct consequence from the flat intensity profile.

Although the recent studies point to a rather large distance to DW UMa, $\geq 500 \mathrm{pc}$, we note that this seems inconsistent with the results of eclipse mapping (Biro 2000; this study). The main source of concern is the relatively high temperature $(\sim 20000 \mathrm{~K})$ at the outer disc regions obtained when distance larger than $500 \mathrm{pc}$ is assumed. To achieve this, a very high mass accretion rate would be needed. It seems not very likely that the reason for this is the usage of a simplified limb-darkening law. The cause of the discrepancy is a puzzle. Although the dependence of limb-darkening on both the temperature and surface gravity is in favor of obtaining flatter radial temperature profiles (compared to if constant limb-darkening coefficient is used), this could hardly explain the very flat profile in DW UMa. The tests of the algorithm over simulated eclipses showed a good 

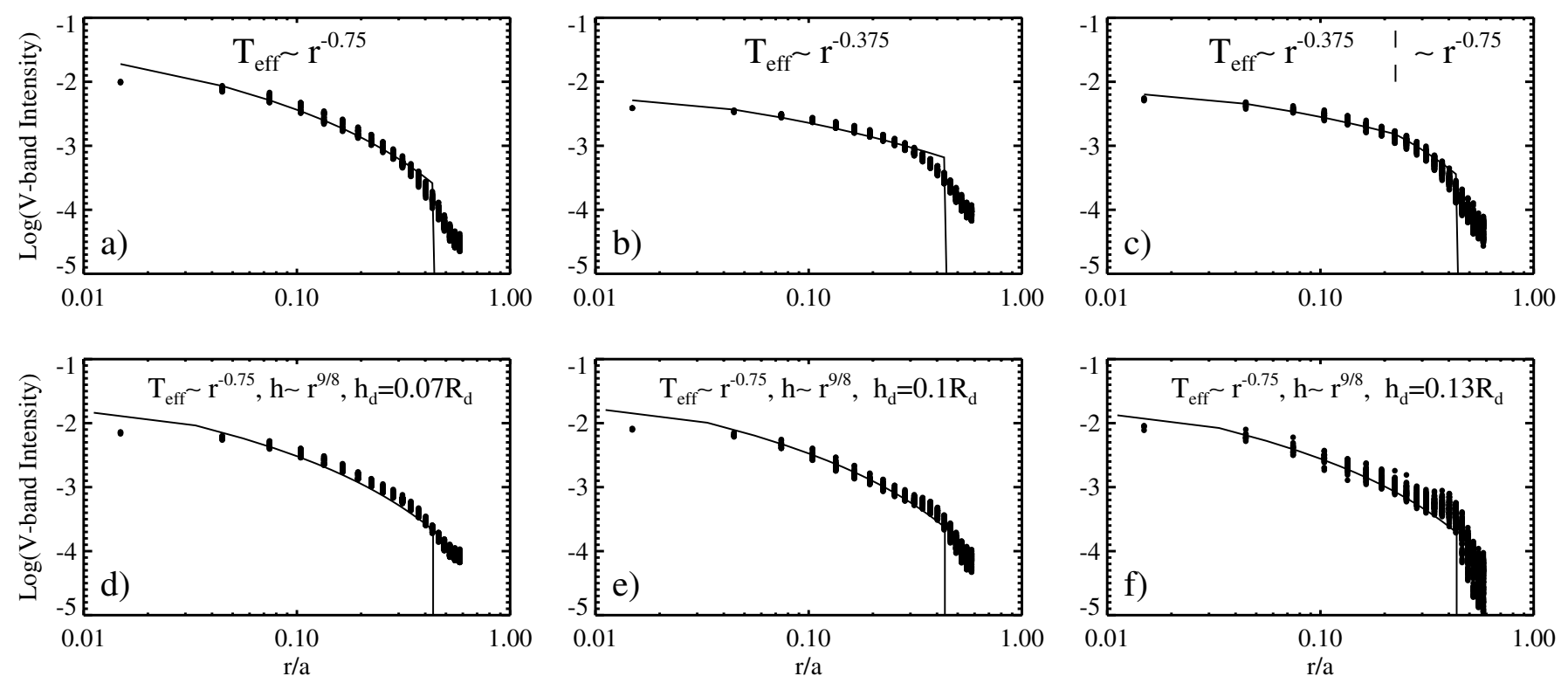

Fig. 8. Reconstruction of the radial $V$-band intensity profile of simulated eclipses with $S / N$ ratio of 100 . Dots - the reconstruction, solid line the disc profile used to simulate the eclipse. a-c) Flat discs with different radial dependence of the temperature, $\mathbf{d}-\mathbf{f}$ ) 3D steady-state discs with emitting outer rims of different height (see text for details). In all reconstructions the eclipses were fitted to reduced $\chi^{2}=1$ using a flat $1 \mathrm{D}$ disc geometry and full azimuthal streaming default images.

recovery of the radial profile. Results of some of the simulations of flat discs are shown in Figs. $8 \mathrm{a}-\mathrm{c}-\mathrm{a}$ ) the $V$-band intensity profile of a steady-state accretion disc with $T_{\text {eff }} \propto r^{-0.75} ; \mathrm{b}$ ) $T_{\text {eff }} \propto r^{-0.375}$ and c) a combination of both. The disc elements are assumed to emits as a black body at given temperature. The emergent radiation is passed through a $V$-band filter, foreshortened and limb-darkened. We used a linear limb-darkening law with a coefficient 0.6 . Gaussian noise of $S / N=100$ was added and so simulated eclipses were fitted to reduced $\chi^{2}=1$ using a full azimuthal streaming default image. It is seen that the recovery in the middle part of the disc is good. Figure 8 also clearly shows one well-known problem of the ME technique, namely that it tends to smear out any sharp structures in the disc. As a result the outer edge of the disc could not be accurately reconstructed and the radial profile is steeper at the outer part. On the other hand, in the inner disc only the very innermost pixels are smoothed and this is not a serious problem for the recovery of the radial profile. We notice that decreasing the noise level to $S / N=300$ does not help much to obtain a better reconstruction and the above situation does not improve significantly.

Of more serious concern are the various systematic effects that may become important in the nearly edge-on systems. As already discussed, the mass accretion rate in NLs is probably not high enough for the disc rim to obscure the disc surface if the inclination is $\sim 80^{\circ}$. However, if $\dot{M} \sim 10^{-8} M_{\odot} \mathrm{yr}^{-1}$ the disc rim may still be as high as $\sim 0.07-0.1 R_{\text {disc }}{ }^{4}$. Recently, Smak (2002) suggested that in this case if the temperature of the rim is comparable to that of the outer disc, in the highinclination NLs the rim may contribute more than $50 \%$ of the system light. Clearly, this would affect the eclipse profile

${ }^{4}$ This refers to the height above the disc mid-plane. The actual size of the rim is twice larger. significantly and when not accounted for may bias the eclipse mapping results.

Smak (1994) and Bobinger et al. (1997) have studied some aspects of the reliability of the reconstruction of 3D discs. Of particular interest is the work of Smak (1994) who tested the reliability of the reconstruction of $3 \mathrm{D}$ discs if a simple flat $1 \mathrm{D}$ disc geometry is used in the reconstruction. Smak's work is however not based on the ME technique. As a part of the testing of our eclipse mapping code we have performed a similar study. We simulated eclipses in a binary with $i=82^{\circ}, q=0.39$, $M_{1}=0.77 M_{\odot}$ and $\dot{M} \sim 10^{-8} M_{\odot} \mathrm{yr}^{-1}$. A steady-state disc, whose (half)thickness varies as $r^{9 / 8}$ is assumed. The outer disc rim is modeled as a cylindrical surface with a temperature equal to that of the outermost part of the disc. The disc has a radius of $0.75 R_{\mathrm{L}_{1}}$. We then simulated eclipses for three values of the rim height, $0.07,0.1$ and $0.13 R_{\text {disc }}$, which are appropriate for very high mass accretion rates. The $V$-band intensities were computed as in the case of the flat disc simulations ${ }^{5}$. Gaussian noise of $S / N=100$ was added and the simulated eclipses were fitted assuming a flat disc geometry.

The reconstructed radial $V$-band intensity profiles are shown in Figs. 8d-f. Generally, our results show that the radial profile of a 3D accretion disc may be still well reconstructed assuming a flat geometry, provided that the inclination is not very high. However, the reconstruction clearly worsens with increasing the rim height and in Figs. 8d-f one can see the increasing contribution of the rim emission. Thus, for very highinclination NLs with $i>85^{\circ}$ this will definitely be a problem,

\footnotetext{
${ }^{5}$ Note that in the disc geometry assumed, the angle at which given disc element is seen is also a function of the orbital phase. In this case the foreshortening and limb-darkening have to be applied simultaneously and they become part of the visibility function (or projection matrix) applied to the disc elements.
} 
especially when the rim stars shielding part of the disc. Note that the impression for a better reconstruction of the disc's edge, compared to the flat disc simulations is misleading. This is due to the additional light from the rim, which compensate for the smoothing of the disc edge. For DW UMa with $i \sim 82^{\circ}$, we may conclude that emission of the outer disc rim cannot be held responsible for the flat intensity profile, unless it is very bright or/and high. Note also that with increasing rim height the scatter of the reconstructed intensities at given radius also increases. The scatter in the maps of DW UMa is nearly twice less than that in the simulation with the rim height $0.13 R_{\text {disc }}$, suggesting that the contribution of the rim in DW UMa is not very significant. We conclude then that the flat temperature profile is not caused by the operation of the algorithm and the assumption of flat disc geometry.

Finally, we would like to note that the SW Sex stars are perhaps the most complex of all types of CVs (Warner 1995). The presence of overflowing stream, possibly a flared accretion disc, accretion disc wind and a magnetic white dwarf violate some of the assumptions of eclipse mapping. In addition, many of the SW Sex stars show superhumps, both "positive" and "negative". The former are now well understood to arise in an elliptic, progradely precessing accretion disc and will certainly complicate the situation ever more. More generally, the presence of hot spots and spiral shocks should also be considered. Detailed investigation of the effect of all these factors on the eclipse mapping is a very complicated task and is out of the scope of this article. We will only briefly discuss some of these topics.

The largest contribution of the spiral shocks should be $\sim 10$ $15 \%$ (e.g. Baptista et al. 2000, 2002). When spread over the disc, this will be much smaller and can hardly affect the radial profile. Pre-eclipse humps similar to those in quiescent dwarf novae are almost never observed in SW Sex stars, suggesting little contribution of the hot spot, if any at all. This is expected though. The traces of the hot spot in the eclipses of dwarf novae disappear in outburst. This generally implies little contribution of the spot when the disc is hot. Because the discs in SW Sex stars are hot, the spot should little affect the eclipse mapping results of these objects. Probably this is also the case with the superhumps, whose light source in NLs contributes no more than $10-20 \%$ of the total system light. A bigger problem with superhumps would be the different disc geometry, for example the elliptic disc shape during the "positive" superhumps. At least in one system, PX And (Stanishev et al. 2002), the eclipse depth varies greatly with the "negative" superhumps phase. This effect may be due to the presence of precessing tilted accretion disc and would have serious implications if the inclination is close to $90^{\circ}$. Thus, both superhumps will affect the eclipse profile. However, when averaging many eclipses observed at different superhump phases, the effect of the superhumps will be reduced and as a result average disc maps will be obtained. Other possible effects such as the spirals being thicker than the rest of the disc and any light scattered in the disc wind or/and the overflowing stream are difficult to take into account and estimate. In conclusion, we may say that various systematic effects may play a role in the eclipse mapping reconstructions of the high-inclination NLs. Thus, the results of such experiments should be always discussed with this in mind.

\section{Summary}

The results of this study can be summarized as follows:

- Photometric observations obtained in intermediate and high states show very large difference in the eclipse depth: $\sim 1.2$ mag in the high and $\sim 3.4$ mag in the intermediate state. Eclipse mapping reveals that this is due to variations of the accretion disc radius resulting in the accretion disc being entirely eclipsed in the intermediate state, whereas in the high state, a large part of the disc remains visible at mid-eclipse. We found that the accretion disc radius increased from $\sim 0.5 R_{\mathrm{L}_{1}}$ in the intermediate state to $\sim 0.75 R_{\mathrm{L}_{1}}$ in the high state $\left(R_{\mathrm{L}_{1}}\right.$ is the distance from the WD to the first Lagrangian point $\left.\mathrm{L}_{1}\right)$;

- The central intensities of the high and intermediate state accretion disc maps are very close. Thus, the increase of the disc radius fully accounts for the final rise after the 1999/2000 low state;

- The accretion disc brightness distribution is very flat. We suggest that this might be due to the presence of a dense wind layer on top of the accretion disc surface. This layer shades inner hot part of the disc and the white dwarf. This simple model may account for: (1) the flat disc profile; (2) the red UV spectrum in high state, and (3) nearly the same temperature of the inner disc in high and intermediate state;

- Based on synthetic stellar spectra we investigate the importance of limb-darkening when calculating the temperature of optically thick accretion discs from eclipse maps. We find that the dependence of limb-darkening on the temperature can have a significant effect on the inferred disc temperatures, and thus should be always taken into account;

- The reliability of the reconstruction of 3D discs if a simple flat $1 \mathrm{D}$ disc geometry is used in the reconstruction is tested. We explored the case of a steady-state disc seen at inclination $i=82^{\circ}$ and possessing an emitting outer rim of different heights. The temperature of the rim is assumed equal to that of the outermost part of the disc. It is concluded that the radial profile of such a disc may still be well reconstructed assuming a flat geometry, provided that the height of the disc rim is less than $\sim 0.1 R_{\text {disc. }}$. If the disc rim is larger than that, the $1 \mathrm{D}$ reconstructed disc shows a larger scatter than is presently observed in DW UMa. The importance of other effects that may introduce systematic errors in the reconstruction of nearly edge-on discs is also discussed;

- Periodogram analysis of the high state data reveals the presence of "positive superhumps" with a period of $P_{\mathrm{sh}}^{+}=$ 0.1455 , in accord with the results of Patterson et al. However, we cannot confirm the quasi-periodic oscillations reported by these authors. In 2003 we also detect "positive superhumps" with a period of $P_{\mathrm{sh}}^{+}=0 \mathrm{~d} \cdot 1461$;

- We obtain an updated orbital ephemeris: $T_{\min }$ [HJD] = $2446229.00687(9)+0.136606527(3) E$. 
In conclusion we want to emphasize the importance of doing time series photometry and spectroscopy of eclipsing SW Sex stars which show low states and particularly during their low/high state transitions. This may give us important clues for understanding their nature and for accretion disc physics in general.

Acknowledgements. HMJB and $\mathrm{CP}$ wish to thank Prof. Wilhelm Seggewiss and Prof. Klaus Reif for generously allocating time at Hoher List. We thank Kent Honeycutt for kindly providing us with his RoboScope photometry of DW UMa in machine readable format. Thierry Pauwels is acknowledged for his help in collecting the data. V. Stanishev acknowledges a grant from the Belgian Federal Office for Scientific, Federal and Cultural Affairs (OSTC) in the frame of the project "Multicolor photometry and astrometry of double and multiple stars". The work was partially supported by NFSR of Bulgaria with the project No. 715/97 and by the IAP P5/36 project of the Belgian Fed. State.

\section{References}

Araujo-Betancor, S., Knigge, C., Long, K. S., et al. 2003, ApJ, 583, 437

Baptista, R., Steiner, J. E., \& Horne, K. 1996, MNRAS, 282, 99

Baptista, R., Horne, K., Wade, R. A., et al. 1998, MNRAS, 298, 1079

Baptista, R., Harlaftis, E. T., \& Steeghs, D. 2000, MNRAS, 314, 727

Baptista, R., Haswell, C. A., \& Thomas, G. 2002, MNRAS, 334, 198

Biro, I. B. 2000, A\&A, 364, 573

Biro, I. B., \& Borkovits, T. 2000, IBVS, 4967

Bobinger, A., Horne, K., Mantel, K.-H., \& Wolf, S. 1997, A\&A, 327, 1023

Borkovits, T., Biro, I. B., \& Kovacs T. 2001, IBVS, 5206

Borkovits, T., Biro, I. B., Hegedus, T., et al. 2002, IBVS, 5313

Borkovits, T., Biro, I. B., Hegedus, T., et al. 2003, IBVS, 5434

Dhillon, V. S., Jones, D. H. P., \& Marsh, T. R. 1994, MNRAS, 314, 826

Diaz, M. P., Wade, R. A., \& Hubeny, I. 1996, ApJ, 459, 236

Harrop-Allin, M. K., \& Warner, B. 1996, MNRAS, 279, 219

Hellier, C. 2000, NewAR, 44, 131
Henden, A. A., \& Honeycutt, R. K. 1995, PASP, 107, 324

Hessman, F. V. 1990, IAU Circ. 4971

Honeycutt, R. K., Turner, G. W., Adams, B. R. 2003, in Interplay of Periodic, Cyclic and Stochastic Variability in Selected Areas of the H-R Diagram ed. C. Sterken, ASP Conf. Ser., 292, 329

Honeycutt, R. K., Livio, M., \& Robertson, J. W. 1993, PASP, 105, 922 Horne, K. 1985, MNRAS, 213, 129

Groot, P. J., Augusteijn, T., Barziv, O., \& van Paradijs, J. 1998, A\&A 340, L31

Knigge, C., \& Drew, J. 1997, ApJ, 486, 445

Knigge, C., Long, K. S., Hoard, D. W., Szkody, P., \& Dhillon, V. S. 2000, ApJ, 539, L49

Kraicheva, Z., Stanishev. V., Genkov, V., \& Iliev L 1999a, A\&A, 351, 607

Kraicheva, Z., Stanishev, V., \& Genkov, V. 1999b, A\&AS, 134, 263

Marsh, T. R., \& Dhillon, V. S. 1997, MNRAS, 292, 385

Patterson, J., Fenton, W. H., Thorstensen, J. R., et al. 2002, PASP, 114, 1364

Rodriguez-Gil, P., Casares, J., Martinez-Pais, I. G., \& Hakala, P. 2002 in The Physics of Cataclysmic Variables and Related Objects, ed. B. T. Gansicke, K. Beuermann, K. Reinsch, ASP Conf. Proc., 261, 533

Rodriguez-Gil, P., Casares, J., Martinez-Pais, I. G., Hakala, P., \& Steeghs, D. 2001, ApJ, 548, L49

Roberts, D., Lehár, J., \& Dreher, J. 1987, AJ, 93, 963

Rutten, R. G. M., van Paradijs, J., \& Tinbergen, J. 1992, A\&A, 260, 213

Shafter, A. W., Hessman, F. V., \& Zhang, E.-H. 1988, ApJ, 327, 248

Stetson, P. 1987, PASP, 99, 191

Skilling, J., \& Bryan, R. K. 1984, MNRAS, 211, 111

Smak, J. 1994, AcA, 44, 265

Smak, J. 2002, AcA, 52, 263

Stanishev, V., Kraicheva, Z., Boffin, H. M. J., \& Genkov, V. 2002, A\&A, 394, 625

Thorstensen, J. R., Ringwald, F. A., Wade, R. A., Schmidt, G. D., \& Norsworthy, J. E. 1991, AJ, 102, 272

Wade, R. A., \& Rucinski, S. M. 1985, A\&AS, 60, 471

Warner, B. 1995, The cataclysmic variables stars (Cambridge University Press)

Wood, J., Abbott, T., \& Shafter, A. 1992, ApJ, 393, 729 